\title{
Proximity and generalized uniformity
}

by

E. M. Alfsen and O. Njåstad (Oslo)

In his paper [11], p. 56i, Yu. M. Smirnor raises the problem whether every proximity structure admits a finest uniform structure compatible with it. In the terminology of [2], p. 97, the problem concerns the existence of a finest uniform structure within every $p$-equivalence class. This question gains particular interest from the fact that such a finest structure would determine a minimal completion among those determined by uniform structures of a given $p$-class.

In another paper [12], p. 764, Smirnor claims that the answer to the problem is negative and sketches a counter-example, which he later ([15], p. 1282) proves to be erroneous. Up to now the question has been open, as far as we know.

In the paper [12], p. 761, Smirnor defines the concept of a complete proximity space without reference to any particular uniform structure, and he proves that erery proximity space admits a minimal completion of this kind. Further investigations along the same lines have been performed by S. Mrówka ([9], [10]) and S. Leader ([7], [8]). Thus the general existence of a minimal completion is established, but the 1-1 correspondence between uniform structures and completions has been lost.

In Section 1 of the present paper we give an (as we believe correct) example of a proximity class of uniform structures without any finest member.

In Section 2 we introduce a generalized notion of uniform structure by replacing the intersection-axiom for entourages by a less restrictive axiom which only involves uniform neighbourhoods of sets and not the entourages themselves. Our discussion is primarily based on A. Weil's axioms ([16], p. 8, [4], p. 131), but in an appendix to the paper, we sketch how one could start out from Smirnov's foundations in terms of coverings ([11], p. 572). Then the passage to generalized uniform structures would consist in the replacement of a "general intersection-axiom" by a "finite intersection-axiom". After some introductory remarks we prove that for generalized uniform structures the answer to Smirnovs problem is affir- 
mative, and we give an explicite characterization of those generalized uinform structures which occur as the finest member of their respective $p$-classes. They are the total structures defined in $\$ 2$, and they woll play a role in the theory which is in a certain sense dual to the role played by the totally bounded structures. We also show that the class of total generalized uniform structures comprises all ordinary metrizable (or pseudo-metrizable) uniform structures. The proof of this statement is based on a lemma of Efremovič ([6], p. 190), and the theorem itself is in fact only a restatement of Smirnov's Theorem 19 of [11], p. 570.

In Section 3 we point out that the $p$-equivalence relation between generalized uniform structures is compatible with the complete latticestructure on the collection of generalized uniform structures, a result which has no counterpart in the theory of proper (i.e. not generalized) uniform structures (cf. the example of $\S 1$ ). By means of this result we obtain new information concerning the interrelationship between proximity continuity and uniform continuity. In particular we show that Efremoriě's theorem on the equivalence of metric uniform continuity and metric proximity continuity ([6], p. 190), as well as the equivalence of totally bounded uniform continuity and proximity continuity, proved in [1], p. 357, both are specializations of the general characterization of proximity continuity by means of uniform continuity.

In Section 4 we prove that every generalized uniform space can be completed and also that the appearence of new completions corresponding to non-proper uniform structures will reestablish the 1-1 correspondence between (generalized) uniform strucutres and (proximity space) completions. Hence there is no more need for an independent treatment of the latter. In particular, the minimal completion of a proximity space (by Smirnov denoted the completion [12], p. 761) is obtained by completion of the finest generalized structure compatible with the proximity structure.

\section{A $p$-eqivalence class of uniform structures not pos-} sessing any finest member. We shall need an auxilliary result to which we shall also make references in the subsequent sections. It is related to Lemma 1 of [11], p. 568 .

LEMMA. Let $\chi_{\omega}$ be the (totally bounded) coarsest uniform structure of a p-equivalence class $P$ of uniform structures on a set $S$. If $V$ is a subset of $S \times S$ for which:

$$
A \Subset V(A)(\mathscr{P}), \text { for all } A \subset S,
$$

then we also have

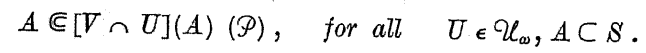

Proof. We may assume $U=\bigcup_{i=1}^{n}\left(\mathcal{A}_{i} \times A_{i}\right)$ where $\left\{A_{i}\right\}_{i=1, \ldots, n}$ is a $p$-covering of $S$ (conf. [1], p. 354, Theorem 1). First we verify (1.2) for $n=2$, and then we proceed by induction.

1. For $n=2$ we hare

$$
\begin{aligned}
{[V \cap U](A) } & =[V \cap U]\left(A-A_{2}\right) \cup[\nabla \cap U]\left(A-A_{1}\right) \cup[V \cap U]\left(A \cap A_{1} \cap A_{2}\right) \\
& =\left[\nabla\left(A-A_{2}\right) \cap A_{1}\right] \cup\left[\nabla\left(A-A_{1}\right) \cap A_{2}\right] \cup V\left(A \cap A_{1} \cap A_{2}\right) .
\end{aligned}
$$

By use of distributivity and after some reduction, this transforms to

(1.3) $[\nabla \cap U](A)=\nabla(A) \cap\left[A_{1} \cup \nabla\left(A \cap A_{2}\right)\right] \cap\left[A_{2} \cup \nabla\left(A \cap A_{1}\right)\right]$.

Now we observe that the following relations hold:

$$
\begin{aligned}
& A-A_{2} \subset \mathrm{C} A_{2} \Subset U\left(C A_{2}\right)=A_{1}, \\
& A \cap A_{2} \Subset V\left(A \cap A_{2}\right) .
\end{aligned}
$$

Combining these two statements by means of elementary properties of the relation "") (cf. formula (1.4) of [1], p. 354) we obtain:

$$
A=\left(A-A_{2}\right) \cup\left(A \cap A_{2}\right) \Subset A_{1} \cup V\left(A \cap A_{2}\right) \text {. }
$$

Similarly we prove:

$$
A \Subset A_{2} \cup V\left(A \cap A_{1}\right) .
$$

Applying these two relations to (1.3) and using elementary properties of the relation "ㄷ" ", we obtain the desired conclusion:

$$
A \Subset[V \cap U](A) \text {. }
$$

2. For $n>2$ we apply the formula of [3] by which we can write

$$
U=\bigcap_{i=1}^{N} U_{i}
$$

where $U_{i}=\left(B_{1}^{i} \times B_{1}^{i}\right) \cup\left(B_{2}^{i} \times B_{2}^{i}\right)$ and $\left\{B_{1}^{i}, B_{2}^{i}\right\}$ is a $p$-covering for every $i$, $i=1, \ldots, N$, and $N=2^{n}$.

Proceeding by induction with respect to $N$ and making use of the statement just proved, we obtain the validity of the general formula (1.2), q.e.d.

THeOREM 1. Let $S$ be a set admitting two partitions $\left\{A_{i}\right\}_{i=1,2, \ldots}$, $\left\{B_{j}\right\}_{j=1,2, \ldots}$ such that $A_{i} \cap B_{j} \neq \varnothing$ for $i, j=1,2, \ldots$, and let $\mathcal{U}, \vartheta$ be the uniform structures defined by the single entourages $U=\bigcup_{i=1}^{\infty}\left(A_{i} \times A_{i}\right)$ and $\nabla=\bigcup_{j=1}^{\infty}\left(B_{j} \times B_{j}\right)$, respectively. Then there is no finest uniform structure in the $p$-class of $\sup \left(\mathcal{U}_{\omega}, \mathfrak{V}_{\omega}\right)$. (For the definition of $\mathcal{U}_{\omega}, \mathcal{V}_{\omega}$, see [1], p. 354.) 
Proof. 1. We first prove that $\sup (\mathcal{U}, \mathcal{V})$ is of a strictly finer $p$-class than $\sup \left(\mathcal{U}_{\omega}, \mathcal{V}_{\omega}\right)$. (For the definition of "finer", resp. "coarser" $p$-class, cf. [2], p. 102.)

The structure $\sup (\mathcal{U}, \mathcal{V})$ is defined by the single entourage

$$
U \cap \nabla=\bigcup_{i, j=1}^{\infty}\left[\left(A_{i} \cap B_{j}\right) \times\left(A_{i} \cap B_{j}\right)\right]
$$

Using the explicite characterization ([1], p. 354) of $\mathcal{U}_{\omega}$ and $\mathcal{V}_{\omega}$, we conclude that $\sup \left(\mathcal{U}_{\infty}, \mathcal{V}_{\infty}\right)$ has a fundamental system of entourages of the form

$$
W=\bigcup_{\substack{m=1, \ldots, M \\ n=1, \ldots, N}}\left[\left(E_{m} \cap F_{n}\right) \times\left(E_{m} \cap F_{n}\right)\right]
$$

where $\left\{\boldsymbol{E}_{m}\right\}_{m=1, \ldots, M}$ and $\left\{\boldsymbol{F}_{n}\right\}_{n=1, \ldots, N}$ are such finite partitions of $S$ that each $E_{m}$ is a union of sets $A_{i}$, and each $F_{n}$ is a union of sets $B_{j}$.

Now we consider the set

$$
G=\bigcup_{k=1}^{\infty}\left[\left(\bigcup_{l=1}^{k} A_{l}\right) \cap B_{k}\right] .
$$

Evidently the relation $G \Subset G$ subsists relatively to $\sup (\mathcal{U}, \mathcal{V})$. We shall prove that the similar relation is inexact relatively to $\sup \left(\mathfrak{U}_{\omega}, \vartheta_{\omega}\right)$.

Let $W$ be any entourage of the type (1.6). Among the sets $E_{m}$ there is at least one, say $E_{m_{0}}$, which contains more than one $A_{i}$ as a subset. In particular, let $A_{i_{1}} \subset E_{m_{0}}, A_{i_{2}} \subset E_{m_{0}}$ and $i_{1}<i_{2}$. Among the sets $F_{n}$ there is just one, say $F_{n_{0}}$, which contains $B_{i_{1}}$.

Let $x \in A_{i_{1}} \cap B_{i_{1}}$ and $y \in A_{i_{2}} \cap B_{i_{1}}$. Then $x \in G, y \in \mathrm{C} G$ and

Thus

$$
(x, y) \in\left[\left(E_{m_{0}} \cap E_{n_{0}}\right) \times\left(E_{m_{n}} \cap F_{n_{0}}\right)\right] \subset W .
$$

$$
y \in \mathrm{C} G \cap W(G) \text {. }
$$

Since $W$ was arbitrary, this means that $G \notin G$ relatively to $\sup \left(\mathcal{U}_{\omega}, \vartheta_{\infty}\right)$.

2. We assume that the $p$-class of $\mathcal{W}=\sup \left(\mathcal{U}_{\omega}, \mathcal{V}_{\omega}\right)$ possesses a finest uniform structure $\mathcal{W}^{\prime}$, and we consider the structures

$$
\mathcal{U}^{\prime}=\sup \left(\mathcal{U}, \mathcal{V}_{\omega}\right)=\sup (\mathcal{U}, \mathcal{W}), \quad \mathcal{V}^{\prime}=\sup \left(\mathcal{V}, \mathcal{U}_{\omega}\right)=\sup (\mathcal{W}, \mathcal{W}) .
$$

Clearly $\mathcal{U}^{\prime}, \mathcal{V}^{\prime}$ are of $p$-classes finer than $\mathcal{W}$, and it follows by the lemma that they are also of $p$-classes coarser than $\mathcal{W}$. Hence $\mathcal{U}^{\prime}$ and $\mathcal{V}^{\prime}$ are both of the same class as $\mathcal{W}$. Thus they are coarser than $\mathcal{W}^{\prime}$, and so $\sup \left(\mathcal{U}^{\prime}, \mathcal{V}^{\prime}\right)$ is coarser than $\mathcal{W}^{\prime}$. Hence $\sup \left(\mathcal{U}^{\prime}, \mathcal{V}^{\prime}\right)$ is of the same class as $\mathcal{W}$ and $W^{\prime}$.

On the other hand

$$
\sup \left(\mathcal{U}^{\prime}, \mathcal{V}^{\prime}\right)=\sup (\mathcal{U}, \mathcal{V})
$$

Hence $\sup \left(\mathcal{U}^{\prime}, \mathcal{V}^{\prime}\right)$ should be of a strictly finer $p$-class than $\mathcal{W}^{\prime}$, in virtue of the first part of the theorem. This contradiction accomplishes the proof.

2. Generalized uniform structures. We shall study collections $\vartheta$ of sets $V \subset S \times S$ satisfying the customary requirements to uniform structures except the intersection axiom which is replaced by a less restrictive condition involving only intersections of uniform set-neighbourhoods $\nabla(A)$. Nore specifically, the collection $\vartheta$ of entourages $T$ of a generalized uniform structure satisfies the following requirements:

(G.U.1) $1=\{(x, x) \mid x \in S\} \subset \underset{\nabla \in \mathcal{Y},}{\subset} V$,

(G.U.2) $U \in \mathcal{V}, U \subset V \Rightarrow V \in \mathcal{V}$.

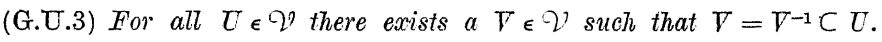

(G.U.4) For all $U \in \mathcal{V}$ there exists a $V \in \mathcal{V}$ such that $V^{2} \subset U$.

(G.U.5) If $A_{1}, \ldots, A_{n} \subset S$ and $U_{1}, \ldots, U_{n} \in \mathcal{V}$ are arbitrary, then there exists a single entourage $U \in \mathcal{V}$ such that

$$
U\left(A_{i}\right) \subset U_{i}\left(A_{i}\right) \quad \text { for } \quad i=1, \ldots, n \text {. }
$$

The relation $A \subset B$ is defined in the usual way (cf. [1], p. 353), and it is easily seen to satisfy the requirements (P.1)-(P.6) of [1], p. 354 . Hence we conclude:

Proposition 1. The generalized uniform structures determine proximity structures and hence also topologies in the same way as (proper) uniform structures do. The topology associated with a generalized uniform structure is completely regular whenever the structure is separating in the sense of

(G.U.1') $\quad \Delta=\bigcap_{V \in \Upsilon^{\prime},} \nabla$.

Now we may define the relation of $p$-equivalence for generalized structures in the same way as for (proper) uniform structures ([2], p. 97). In the sequel we shall use the term " $p$-equivalence class" or briefly " $p$-class" to mean a $p$-equivalence class of generalized uniform structures, unless otherwise is stated. We also define the order relation ("finer", "coarser") between generalized uniform structures in the customary way.

A generalized uniform structure $\mathscr{U}$ on $S$ will be said to be totally bounded if the entire space $S$ is a totally bounded set with respect to $\mathcal{U}$, i.e. if it is possible for every entourage $V$ of $\mathcal{U}$ to find a finite covering $\left\{A_{i}\right\}_{1 \leqslant i \leqslant n}$ of $S$ such that $A_{i} \times A_{i} \subset V, i=1, \ldots, n$ (cf. [1], p. 356).

The Theorems 1, 2 of [1] remain valid for generalized uniform structures. More specifically, we shall have the following 
THEOREM 2. Every p-equivalence class of generalized uniform structures contains a coarsest member $\varkappa_{\omega}$ which is a proper uniform structure with a base consisting of the sets:

$$
V=\bigcup_{i=1}^{n}\left(A_{i} \times A_{i}\right)
$$

where $\left\{A_{i}\right\}_{1 \leqslant i \leqslant n}$ is some $p$-covering of $S$ (cf. [1], p. 353).

Furthermore $\mathscr{U}_{\omega}$ is the only totally bounded, generalized uniform structure of the class.

Proof. 1. It follows from Theorems 1, 2 of [1] that the sets (2.1) form a base of a totally bounded uniform structure which is the coarsest proper uniform structure of the class. We shall prove that $\mathcal{U}_{\omega}$ is coarser than any generalized uniform structure $U$ of the class as well.

Let $\nabla$ be some set of the form (2.1). By the definition of a $p$-covering there exist sets $B_{i}, i=1, \ldots, n$, such that $B_{i} \Subset A_{i}$ for $i=1, \ldots, n$ and $S=\bigcup_{i=1}^{n} B_{i}$. Since $\mathcal{U}$ is supposed to be a member of the given $p$-class, there exist entourages $U_{i}, i=1, \ldots, n$, of $\nVdash$ such that $U_{i}\left(B_{i}\right) \subset A_{i}$ for $i=1, \ldots, n$. In virtue of axiom (G.U.5) there exists a single entourage $U$ of $\chi$ such that $U\left(B_{i}\right) \subset A_{i}$ for $i=1, \ldots, n$.

Hence:

$$
U \subset \bigcup_{i=1}^{n}\left[U\left(B_{i}\right) \times U\left(B_{i}\right)\right] \subset \bigcup_{i=1}^{n}\left(A_{i} \times A_{i}\right)=V .
$$

Thus we have proved $\mathcal{U}$ to be finer than $\varkappa_{\omega}$, q.e.d.

2. To prove $\mathcal{U}_{\omega}$ to be the only totally bounded generalized structure of the class, we may proceed as in the second part of the proof of Theorem 2 of $[1]$, since the intersection axiom of proper uniform structures was not involved in that proof.

COROLLARY. If a generalized uniform structure is totally bounded, then it is necessarily a proper uniform structure.

The introduction of generalized uniform structures enables us to obtain a new result which is in a certain sense a dual to Theorem 2. To facilliate the formulation and proof of this result, we introduce the following notation: A subset $V$ of $S \times S$ is said to be entourage-like relatively to a proximity structure on $S$ if it admits a sequence $\left\{V_{n}\right\}_{n=1,2, \ldots}$ of symmetric subsets of $S \times S$ such that:

$$
\begin{array}{rlr}
\text { (2.2) } \quad \nabla_{1}^{2} \subset V, & \nabla_{n+1}^{2} \subset \nabla_{n}, \quad A \Subset \nabla_{n}(A) \quad \text { for all } \quad A \subset S \\
& \text { and } n=1,2, \ldots
\end{array}
$$

We shall say that a subset $V$ of $S \times S$ is entourage-like relatively to a proximity class of generalized uniform structures, or relatively to a single such structure, if it is entourage-like relatively to the associated proximity structure. Clearly every entourage of a generalized uniform structure is entourage-like. Generalized uniform structures for which the reverse statement holds, i.e. for which every entourge-like set is an entourage, will be said to be total.

THEOREM 3. Every $p$-equivalence class of generalized uniform structures contains a finest member $\chi_{a}$, and the entourages of $\chi_{a}$ are exactly the entourage-like sets of the given $p$-class. $B y$ definition, $\chi_{\alpha}$ is the only total structure of the class.

Proof. Let $\mathcal{V}$ be the collection of all entourage-like sets relatively to the given $p$-class. Clearly, every entourage $V$ of $\chi_{\omega}$ belongs to $\urcorner$, and $\vartheta$ satisfies the requirements (G.U.1)-(G.U.4). To prove that $\vartheta$ satisfies (G.U.5), we assume $A_{1} \ldots A_{n} \subset S$ and $U_{1} \ldots U_{n} \in \mathcal{V}$. By the definition (2.2) we conclude that $A_{i} \Subset U_{i}\left(A_{i}\right)$ for $i=1, \ldots, n$. Being a member of the $p$-class in question, $\chi_{\omega}$ must contain entourages $\nabla_{i}, i=1, \ldots, n$, such that $\nabla_{i}\left(A_{i}\right) \subset U_{i}\left(A_{i}\right)$ for $i=1, \ldots, n$. Now $\nabla=\bigcap_{i=1}^{n} \nabla_{i}$ is still an. entourage of the proper uniform structure $\chi_{\omega}$. Hence $V \epsilon \mathcal{V}$, and so the relations $V\left(A_{i}\right) \subset U_{i}\left(A_{i}\right)$ for $i=1, \ldots, n$ yield the validity of (G.U.5).

Thus we have proved $\mathcal{Y}$ to be the collection of entourages of a generalized uniform structure $\ell_{\omega}$ which clearly must be of a $p$-equivalence class finer than the given class. It follows from the definition (2.2) that $\mathfrak{\ell}_{a}$ also is of a $p$-class coarser than the given class, and hence it must belong to it. $\mathscr{U}_{\alpha}$ is obviously the finest member of this class since every entourage of any other member must be entourage-like, and so belong to $\mathcal{\nu}$.

If the collection of entourages of a generalized uniform structure $\mathcal{U}$ is the union of the collections of entourages of a family $\left\{\mathcal{U}_{\gamma}\right\}_{\gamma \in \Gamma}$ of structures, then we shall simply say that $\mathcal{U}$ is the union of $\left\{\mathcal{U}_{\gamma}\right\}_{\gamma \in \Gamma}$. A uniform structure which can be defined by means of a single pseudo-metric ("écart" in the terminology of [5], p. 9), will be termed pseudo-metrizable. It is classical that a uniform structure is pseudo-metrizable if and only if it admits an enumerable base. ([5], p. 15).

THEOREM 4. The finest generatized structure $\varkappa_{\alpha}$ of a given $p$-equivalence class $\mathcal{P}$ is equal to:

(1) The union $\mathscr{U}_{1}$ of all generalized uniform structures of the class $\mathcal{P}$.

(2) The union $\varkappa_{2}$ of all generalized uniform structures of classes coarser than $P$.

(3) The union $\chi_{3}$ of all proper uniform structures of the class $P$.

(4) The union $\varkappa_{4}$ of all proper uniform structures of classes coarser than $P$.

(5) The union $\mathcal{U}_{5}$ of all psendo-metrizable uniform structures of classes coarser than $P$. 
Proof. 1. Using the set-theoretic inclusion sign with the obvious meaning, we can write:

$$
\varkappa_{\alpha} \subset \varkappa_{1} \subset \varkappa_{2} \subset \varkappa_{\alpha}
$$

The first relation follows from the fact that $\varkappa_{a}$ itself is a generalized uniform structure of the class $\mathcal{P}$. The second relation is trivial, and the third relation follows from the fact that every entourage of any structure of a class coarser than $\mathcal{P}$ must be entourage-like relatively to $P$.

2. Leaving aside the less trivial characterization (3) for a moment, we can write:

$$
\mathfrak{U}_{a} \subset \mathfrak{U}_{5} \subset \mathfrak{U}_{4} \subset \mathfrak{U}_{\alpha} .
$$

The first relation follows from the fact that if $V$ is an entourage-like set with a defining sequence $\left\{\nabla_{n}\right\}_{n=1,2, \ldots}$ (cf. (2.2)), then the sets $V_{n}$ will form a base of a pseudo-metrizable uniform structure for which $V$ is an entourage. The remaining two relations are trivial.

3. Trivially $\mathfrak{U}_{3} \subset \mathfrak{U}_{\alpha}$. To prove the reverse relation, we assume $V$ to be some entourage of $\varkappa_{\alpha}$. By the characterization (4), there exists some proper uniform structure $\mathcal{W}$ of a $p$-class coarser than $\mathcal{P}$, such that $T$ is an entourage of $\mathcal{W}$.

Since $\mathcal{W}$ is of a class coarser than $\mathcal{P}$, we have

$$
A \Subset W(A)(\mathscr{P}),
$$

whenever $A \subset S$, and $W$ is an entourage of $\%$.

Now we consider $\mathcal{U}^{\prime}=\sup \left(\mathcal{W}, \mathcal{U}_{\omega}\right)$, i.e. the coarsest proper uniform structure finer than both $\mathcal{W}$ and $\varkappa_{\omega}$. (Here $\mathcal{U}_{\omega}$ is defined as in Theorem 2 relatively to the class $\mathcal{P}$ in question.) In virtue of the lemma to Theorem 1 and the formula (2.3) we shall have:

$$
A \Subset[U \cap W](A)(P),
$$

whenever $A \subset S, U$ is an entourage of $\varkappa_{\omega}$, and $W$ is an entourage of $\vartheta^{\circ}$. This means that $\mathcal{U}^{\prime}$ is of a coarser $p$-class than $\mathcal{P}$. But as $\mathcal{U}^{\prime}$ is finer than $\mathcal{U}_{\omega}$, it is also of a finer class than $\mathcal{P}$, and so $\mathcal{U}^{\prime} \in \mathcal{P}$. Hence $\mathcal{P}$ contains a proper uniform structure, $\mathcal{U}^{\prime}$, for which $V$ is an entourage, and so we have proved $\mathcal{U}_{a} \subset \mathcal{V}_{3}$, q.e.d. (Cf. Theorem 18 of [11].)

COROLLARY 1. If there exists a finest structure among the proper uniform structures of a p-equivalence class, then this structure is equal to $\varkappa_{\alpha}$.

Proof. Application of the characterization (3) of Theorem 4.

COROLIARY 2. There exists a finest structure among the proper uniform. structures of a $p$-equivalence class if and only if its collection of entouragelike sets is closed with respect to finite intersections.
Proof. The condition of the Corollary ensures that $\ell_{\alpha}$ is a proper uniform structure.

COROLLARY 3. There exist total, generalized uniform structures which. are not proper uniform structures.

Proof. The structure $\mathcal{C}_{\alpha}$ of the proximity class of $\sup \left(\mathcal{C}_{\omega}, \vartheta_{\omega}\right)$ in Theorem 1 must be such a structure.

Our next theorem is based on a lemma due to Efremovič ([6], p. 190). For the sake of completeness we sketch the proof. The theorem itself corresponds to Theorem 19 in [11], p. 570.

LEMLA. For a given set $S$ we assume $U$ and $W$ to be such symmetric subsets of $S \times S$ that $W^{4} \subset U$. Then every sequence $\left\{\left(x_{n}, y_{n}\right)\right\}_{n=1,2, \ldots}$ on $S \times S$,. for which $\left(x_{n}, y_{n}\right) \dot{\&} U$ for $n=1,2, \ldots$, admits a subsequence $\left.\left\{x_{n_{k}}, y_{n_{k}}\right)\right\}_{k=1,2, \ldots}$ such that $\left(x_{n_{k}}, y_{n_{l}}\right) \dot{\epsilon} W$ for $k, l=1,2, \ldots$

Proof. For every $n$ we define two sets $B_{n}, C_{n}$ of natural numbers:

$$
B_{n}=\left\{m \mid\left(x_{n}, y_{m}\right) \in W\right\}, \quad C_{n}=\left\{m \mid\left(x_{m}, y_{n}\right) \in W\right\} .
$$

Clearly $\left(y_{p}, y_{q}\right) \in W^{2}$ whenever $p, q \in B_{n}$. Hence we must have $\left(x_{q}, y_{p}\right) \notin W^{2}$ whenever $p, q \in B_{n}$, for otherwise $\left(x_{q}, y_{q}\right) \in W^{4} \subset U$ contrary to the hypothesis. Thus if $B_{n}$ is infinite for any $n$, then $\left.\left\{x_{m}, y_{m}\right)\right\}_{m \in B_{n}}$ is a subsequence with the desired property.

Similarly we prove that if $C_{n}$ is infinite for any $n$, then $\left\{\left(x_{m}, y_{m}\right)\right\}_{m \in C_{n}}$ is a subsequence with the desired property.

Hence we have only to consider the case in which $B_{n}$ and $C_{n}$ are finite for every $n$. Then we denote the first natural number strictly greater than $n$ and all members of all sets $B_{m}$ and $C_{m}, m=1,2, \ldots, n$, by the symbol $\varphi(n)$, and define $n_{1}=1, n_{2}=\varphi\left(n_{1}\right)$, and generally $n_{k+1}=\varphi\left(n_{k}\right)$. Then the sequence $\left\{\left(x_{n_{k}}, y_{n_{k}}\right)\right\}_{k=1,2, \ldots}$ will have the desired property.

THEOREM 5. Every uniform structure $\mathcal{U}$ defined by a single psendometric $\varrho$, is total.

Proof. Let $U$ be an entourage-like set relatively to $U$. By definition there exists another entourage-like set $W$ such that $W^{4} \subset U$.

If $U$ were not an entourage of $\mathcal{~}$, then there would exist a pair of elements $\left(x_{n}, y_{n}\right)$ with the following properties for every $n$ :

$$
\varrho\left(x_{n}, y_{n}\right)<\frac{1}{n}, \quad\left(x_{n}, y_{n}\right) \dot{\epsilon} U .
$$

By the lemma we can extract a subsequence $\left\{\left(x_{n_{k}}, y_{n_{k}}\right)\right\}_{k=1,2, \ldots}$ such that $\left(x_{n_{k}}, y_{n_{l}}\right) \dot{\epsilon} W$ for all $k, l=1,2, \ldots$ Now we define $X=\left\{x_{n_{k}} \mid k=1,2, \ldots\right\}$ $\bar{Y}=\left\{y_{n_{k}} \mid k=1,2, \ldots\right\}$, obtaining $W(X) \subset \mathrm{CY}$, and hence $X \Subset \mathrm{C} Y$.

On the other hand, we may apply the first relation of (2.4), giving $V_{1 / n}(X) \cap Y \neq \varnothing$ for all $n$. (Here $V_{1 / n}$ denotes the entourage consisting of all $(x, y)$ for which $\varrho(x, y)<1 / n$, as usual.) This statement contradicts the relation $X \Subset C Y$, and so the proof is accomplished. 
§ 3. Compatibility of $p$-equivalence and lattice operations for generalized uniform structures. We recall that a proximity class $\mathcal{P}_{1}$ on a set $S$ is said to be coarser than another proximity class $P_{2}$ on $S$ provided the (unique) totally bounded uniform structure $\varkappa_{\omega_{1}}$ associated with $P_{1}$, is coarser than the corresponding structure $\mathcal{U}_{\omega_{2}}$ associated with $P_{2}([2]$, p. 102). This definition corresponds to the similar definition for proximity spaces ([11], p. 557), which simply states that

$$
A \subset B\left(P_{1}\right) \Rightarrow A \Subset B\left(P_{2}\right) \text {. }
$$

From the above definition we immediately deduce the following:

Proposition 2. The collection of $p$-classes on a set $S$ is organized to a complete lattice by the order relation "finer-coarser".

The lattice-supremum of a family $\left\{P_{\gamma}\right\}_{\gamma \in \Gamma}$ of $p$-classes on $S$ will be denoted by the customary symbol $\bigvee_{\gamma \in T} P_{\gamma}$. Similarly we denote the latticeinfinium by the symbol $\bigwedge_{\gamma \in \Gamma} P_{\gamma}$. For the lattice operations on the collection of proper uniform structures we keep the notations $\sup _{\gamma \in \Gamma} \mathcal{U}_{\gamma}, \inf _{\gamma \in \Gamma} \varkappa_{\gamma}$, reserving the symbols $\underset{\gamma \in \Gamma}{\bigvee} \mathcal{U}_{\gamma}, \bigwedge_{\gamma \in \Gamma} \mathcal{U}_{\gamma}$ for the corresponding operations on the collection of generalized uniform structures. (For the existence and explicite characterization of the latter operations, cf. Theorem 6 and the formula (3.2) below.)

Remark to Proposition 2. $\underset{\gamma \in \Gamma}{\bigvee} \mathscr{P}_{\gamma}$ is the $p$-class containing the totally bounded uniform structure $\sup _{\gamma \in \Gamma} \mathcal{U}_{\omega_{\gamma}}$ where $\varkappa_{\omega_{\gamma}}$ denotes the totaly bounded uniform structure associated with $\mathscr{P}_{\gamma}$, for each $\gamma \in \Gamma$. An explicite characterization of $\bigvee_{\gamma \in \Gamma} \mathcal{P}_{\gamma}$ in terms of the relation "드" may be obtained from the formula $(2.6)$ of [1], p. 359. However, we shall not need such a characterization in the sequel.

THEOREM 6. The collection of generalized uniform structures on a set $S$ is organized to a complete lattice by the order relation "finer-coarser", and the p-equivalence-relation is compatible with this lattice structure in the sense that $\bigvee_{\gamma \in \Gamma} \mathcal{U}_{\gamma}\left(\right.$ resp. $\left.\bigwedge_{\gamma \in \Gamma} \mathcal{U}_{\gamma}\right)$ is of the $p$-class $\bigvee_{\gamma \in \Gamma} \mathscr{P}_{\gamma}\left(\right.$ resp. $\left.\bigwedge_{\gamma \in \Gamma} \mathcal{P}_{\gamma}\right)$ whenever $\mathcal{U}_{\gamma}$, $\gamma \in \Gamma$, are generalized uniform structures of $p$-classes $\stackrel{\gamma}{\gamma}, \gamma_{\gamma}, \gamma \in \Gamma$.

Proof. Let $\chi_{\gamma}, \gamma \in \Gamma$ be generalized uniform structures of $p$-classes $\mathscr{P}_{\gamma}$, $\gamma \in \Gamma$, and let $\chi_{\omega}$ denote the totally bounded uniform structure of the $p$-class $\mathcal{P}=\bigvee_{\gamma \in \Gamma} P_{\gamma}$. Let $\mathcal{Q}$ denote the set-theoretic union of the systems of entourages of all $\varkappa_{\gamma}, \gamma \in \Gamma$ and of $\varkappa_{\omega}$. We shall prove that $\mathcal{W}^{3}$ is the system of entourages of a generalized uniform structure which is the coarsest structure finer than all $\varkappa_{y}, \gamma \in \Gamma$.
Evidently the requirements (G.U.1)-(G.U.4) are satisfied. To verify (G.U.5) we assume $A_{i} \subset S$ and $U_{i} \in \mathcal{W}^{0}$ for $i=1, \ldots, n$. For every fixed $i$ the subset $U_{i}$ of $S \times S$ will either be an entourage of some $\chi_{\gamma}, \gamma \in \Gamma$, or of $\varkappa_{\omega}$. Since the structures $\chi_{\gamma}, \gamma \in \Gamma$, are all of $p$-classes coarser than $P$, we shall have $A_{i} \Subset U_{i}\left(A_{i}\right)$ relatively to $\mathcal{P}$ in any case. Consequently there exist entourages $\nabla_{i}$ of $\chi_{\omega}$ such that $\nabla_{i}\left(A_{i}\right) \subset U_{i}\left(A_{i}\right)$ for $i=1,2, \ldots, n$. Now the intersection $V=\Gamma_{1} \cap \ldots \cap \nabla_{n}$ will be an entourage of $\chi_{\omega}$ as well, and hence we have:

$$
\nabla \in \mathcal{W} ; \quad V\left(A_{i}\right) \subset U_{i}\left(A_{i}\right) \quad \text { for } \quad i=1, \ldots, n .
$$

Thus we have proved that $\mathcal{W}$ is the system of entourages of a generalized uniform structure $\mathcal{U}$ on $S$.

The structure $\chi$ is evidently finer than all $\varkappa_{\gamma}, \gamma \in \Gamma$. To see that $\chi$ is coarsest generalized uniform structure with this property, we assume that $\chi^{\prime}$ is some other generalized uniform structure finer than all $\varkappa_{\gamma}$, $\gamma \in \Gamma$, and we only have to prove that $\mathcal{U}^{\prime}$ is finer than $\varkappa_{\infty}$. This however, follows from the fact that $\mathcal{X}^{\prime}$ must be of a $p$-class finer than all $\mathcal{P}_{\gamma}$ and hence finer than $\mathcal{P}$. Thus we can write $\ell=\underset{\nu \in \Gamma}{\bigvee} \mathcal{U}_{\gamma}$.

It follows directly from the definition of $\eta^{\rho}$ that $\chi=\bigvee_{\gamma \in \Gamma} \chi_{\gamma}$ is of the $p$-class $\mathcal{P}=\bigvee_{\gamma \in \Gamma} \mathcal{P}_{\gamma}$.

Finally the existence of $\bigwedge_{\gamma \in \Gamma} \mathcal{U}_{\gamma}$ and the fact that $\bigwedge_{\gamma \in \Gamma} \mathcal{U}_{\gamma}$ is of the $p$-class $\underset{\gamma \in \Gamma}{\wedge} P_{\gamma}$, can be obtained by duality from the statements just proved.

Remark. From the proof of Theorem 6 we conclude that $\underset{\gamma \in T}{\vee} \chi_{\gamma}$ can be expressed explicitely by the following formula in which $\mathscr{U}_{\infty}$ is the totally bounded uniform structure of $\underset{\gamma \in \Gamma}{\bigvee} P_{\gamma}$ (and in which we use the concept of "set theoretic union" of uniform structures in the meaning defined ahead of Theorem 4 ):

$$
\bigvee_{\gamma \in \Gamma} \chi_{\gamma}=\chi_{\omega} \cup \bigcup_{\gamma \in \Gamma} \chi_{\gamma} .
$$

From the compatibility of the $p$-equivalence-relation and the lattice operations for generalized uniform structures, we immediately obtain a series of alternative characterization of the ordering between $p$-classes:

COROLLARY 1. The statement (3.1) is equivalent to each of the following:

(1) For all $\mathcal{U}_{1} \in \mathcal{P}_{1}$ there exists a $\chi_{2} \in \mathcal{P}_{2}$ such that $\mathcal{\ell}_{1}$ is coarser than $\mathcal{L}_{2}$.

(2) There exists a $\mathcal{\ell}_{1} \in \mathcal{P}_{1}$ and a $\mathcal{\ell}_{2} \in \mathcal{P}_{2}$ such that $\mathcal{U}_{1}$ is coarser than $\mathcal{U}_{2}$.

(3) For all $\varkappa_{2} \in P_{2}$ there exists a $\chi_{1} \in P_{1}$ such that $\varkappa_{1}$ is coarser than $\chi_{\ell_{2}}$. 
(4) The finest member $\mathcal{U}_{a_{1}}$ of $\mathcal{P}_{1}$ is coarser than the finest member $\mathscr{C}_{a_{2}}$ of $P_{2}$.

(5) The coarsest member $\mathcal{U}_{\omega_{1}}$ of $\mathcal{P}_{1}$ is coarser than the coarsest member $\mathcal{U}_{\omega_{2}}$ of $P_{2}$.

Proof. The first three statements follow from general characterizations of the order relation for congruence classes modulo a latticecongruence in terms of representatives of the classes. The fourth statement follows from the first statement and implies the second. The fifth statement follows from the third statement and implies the second.

Let $\mathcal{P}_{1}$ and $\mathcal{P}_{2}$ be proximity classes on the sets $S_{1}$ and $S_{2}$, respectively, and let $f$ be a mapping of $S_{1}$ into $S_{2}$. We recall that $f$ is said to be $p$-continuous (w.r. to $P_{1}$ and $P_{2}$ ) if the following implication holds

$$
A \Subset B\left(\mathcal{P}_{2}\right) \Rightarrow f^{-1}(A) \Subset f^{-1}(B)\left(P_{1}\right) .
$$

We now observe that if $\chi$ is a generalized uniform structure on $S_{2}$, then so"is the inverse image by $f$ on $S_{1}$. Hence uniform continuity may be defined for generalized uniform structures in the same way as for proper uniform structures. Clearly the inverse images of $p$-equivalent structures are $p$-equivalent, and the inverse image of a totally bounded structure is totally bounded. By means of Corollary 1 above, we may then give a series of alternative characterizations of $p$-continuity.

CoROLLARY 2. The statement (3.3) is equivalent to each of the following:

(1) For every $\chi_{1} \in P_{1}$ there exists a $\mathcal{l}_{2} \in P_{2}$ such that $f$ is uniformly continuous relatively to $\varkappa_{1}$ and $\varkappa_{2}$.

(2) There exists a $\varkappa_{1} \in \mathcal{P}_{1}$ and a $\mathcal{L}_{2} \in \mathcal{P}_{2}$ such that $f$ is uniformly continous relatively to $\mathcal{U}_{1}$ and $\mathcal{U}_{2}$.

(3) For every $\varkappa_{2} \in \mathcal{P}_{2}$ there exists a $\chi_{1} \in \mathcal{P}_{1}$ such that $f$ is uniformly continuous relatively to $\varkappa_{1}$ and $\varkappa_{2}$.

(4) $f$ is uniformly continuous relatively to the finest structure $\chi_{\alpha_{1}}$ in $\mathcal{P}_{1}$ and the finest structure $\mathcal{U}_{\alpha_{2}}$ in $\mathscr{P}_{2}$.

(5) $f$ is uniformly continuous relatively to the coarsest structure $\mathcal{U}_{\omega_{\mathrm{r}}}$ in $\mathcal{P}_{1}$ and the coarsest structure $\mathfrak{U}_{\omega_{2}}$ in $\mathcal{P}_{2}$.

The statement (5) of Corollary 2 is the characterization given in [1], p. 357, while (4) is essentially that of [8], p. 206. Combining the statement (4) of Corollary 2 with Theorem 5, we obtain the following result which was originally proved by Efremovič as one of the first theorems in the theory of proximity ([6], p. 190).

CoRollari 3. A mapping f from a (pseudo-) metric space into $a$ (pseudo-) metric space is $p$-continuous if and only if it is uniformly continuous.
§ 4. Completion. For the sake of simplicity we restrict ourselves to study separating (cf. (G.T.1')) generalized uniform structures. (For the non-separating case, cf. "Remark" in [2], p. 101.) A set $S$ provided with a generalized uniform structure $\mathcal{U}$ will be termed a generalized uniform space and will be denoted $(S, Z)$. The concepts of a regular filter, Cauchy-filter, complete space, and completion is defined in the customary way (cf. [2], p. 97, [4], p. 145).

We remark that all generalized uniform structures of the same $p$-class will possess the same regular filters, and the Theorems 1,2 of [2] remain valid for generalized structures.

Defining the concept of a base in the obvious way, we can state the following:

Proposimiox 3. Every generalized uniform structure $\mathcal{U}$ on $S$ admits a base of entourages which are closed in the product topology on $S \times S$, when $S$ is provided with the topology associated with $\mathcal{U}$.

The proof is similar to the corresponding proof for proper uniform structures, cf. [4], p. 141. That proof actually involves the intersection of two entourages $U$ and $W$, but only to ensure the existence of a base of symmetric sets. In our case this follows from (G.U.3).

Theorem 7. Let $f$ be a function defined on a dense subset $A$ of a generalized uniform space $(\mathcal{S}, \mathcal{U})$, taking values in a separated, complete, generalized uniform space $\left(S^{\prime}, \mathcal{U}^{\prime}\right)$, and assume $f$ to be uniformly continuous. Then $f$ can be extended to a uniformly continuous function $f$ defined on the entire space $S$.

The proof is similar to the corresponding proof for proper uniform structures and makes use of the existence of a base of closed entourages as established in Proposition 3. For details cf. [4], p. 151.

In the sequel $\mathcal{U}$ shall denote some fixed generalized uniform structure on a set $S$, and we shall use the symbol $S$ to denote the collection of all regular Cauchy-filters of the generalized uniform space $(S, \mathcal{U})$. To every entourage $V$ of $\ell$ we assign a subset $\hat{V}$ of $\hat{S} \times \hat{S}$ defined by the formula: (4.1)

$$
(\mathcal{F}, \mathcal{G}) \epsilon \hat{V} \Leftrightarrow(\mathbb{H} C \in \mathcal{F} \cap \mathcal{G})[C \times O C V] \text {. }
$$

For every Cauchy-filter $\mathcal{F}$ and every entourage $\nabla$ of $\mathcal{U}$ we define the (non-empty) subset $Q_{\mathscr{F}}^{V}$ of $S$ to be the union of all those $C \in \mathcal{F}$ which are small of order $\nabla$ (i.e. for which $C \times O \subset V$ ).

For every subset $\hat{A}$ of $\hat{S}$ and every entourage $\nabla$ of $\mathcal{U}$ we define:

$$
\nabla[\hat{A}]=\bigcup_{\mathscr{F} \in \hat{A}} Q_{\mathcal{F}}^{V} .
$$

Lemoma. If $\nabla, W \in \mathcal{U}, \hat{A} \subset \hat{S}$ and

$$
W(\nabla[\hat{A}]) \subset V(\nabla[\hat{A}])
$$

then

$$
\widehat{W}(\widehat{A}) \subset \widehat{V^{3}}(\hat{A}) \text {. }
$$


Proof. Let $\mathcal{F} \epsilon \widehat{W}(\hat{A})$, i.e. there exists a $\mathcal{G} \in \hat{A}$ and a $C \in \mathcal{F} \cap \mathcal{G}$ such that $C \times C \subset W$.

The filter $Q$ will contain a set $B$ which is small of order $V$ and is a subset of $C$. Hence $B \subset Q_{\mathcal{E}}^{V}, B \subset C$. By the definition (4.2),

$$
B \subset V[\hat{A}] .
$$

Let $x \in C$ and $y \in B \subset C$ be arbitrary. Then $(x, y) \in C \times C \subset W$, and so $x \in W(B)$. Hence we have $C \subset W(B)$, and so:

$$
C \subset W(B) \subset W(V[\hat{A}]) \text {. }
$$

By the assumption of the Lemma this entails

$$
C \subset V(\nabla[\hat{A}]) \text {. }
$$

Let $p, q$ be two points of $S$ such that:

$$
p \in \nabla[\hat{A}], \quad q \in \nabla\left[C \widehat{V}^{3}(\hat{A})\right]
$$

By the definition (4.2), there exist subsets $D_{p}, D_{q}$ of $S$ small of order $\nabla$ such that $p \in D_{p}, q \in D_{q}$, and such that $D_{p}$ belongs to some filter $\mathcal{C}_{p}$ of $\hat{A}$ and $D_{q}$ belongs to some filter $\mathscr{H}_{q}$ which is not in $\widehat{V}^{3}(\hat{A})$. Hence $\left(\mathscr{H}_{p}, \mathscr{H}_{q}\right) \notin \widehat{\nabla^{3}}$. Thus we have $(p, q) \dot{\epsilon}$, since otherwise $D_{p} \cap D_{q}$ would belong to $\mathscr{H}_{p} \cap \mathscr{H}_{q}$ and be small of order $\nabla^{3}$, giving $\left(\mathscr{H}_{p}, \mathscr{H}_{q}\right) \in \widehat{V^{3}}$. Otherwise stated, $q \dot{\epsilon}(p)$, and so we have verified that:

$$
V\left[C \nabla^{3}(\hat{A})\right] \cap V(\nabla[\hat{A}])=\varnothing .
$$

By the definition (4.2), this means that $V(\nabla[\hat{A}])$ admits no subset $D$ small of order $V$, belonging to any $\mathcal{H} \dot{\epsilon} \widehat{V^{3}}(\widehat{A})$. Hence we have proved the implication

$$
\text { (4.4) } \quad D \times D \subset V, \quad D \subset \nabla(\nabla[\hat{A}]), \quad D \in \mathcal{H} \in \hat{S} \Rightarrow \mathscr{H} \in \widehat{\nabla}^{3}(\hat{A}) \text {. }
$$

The original filter $\mathcal{F}$ contains a set $D$ such that $D \times D \subset V$ and $D \subset C$. By (4.3) we have $D \subset V(\nabla[\hat{A}])$, and hence by (4.4) $\mathscr{F} \epsilon \widehat{V^{3}}(\hat{A})$, q.e.d.

We are now able to state and prove the completion theorem for generalized uniform spaces in a form which brings out clearly the relations to the compactifications of the associated proximity spaces (Cf. [2], p. 103, [11], p. 556.)

THEOREM 8. Let $\mathcal{U}$ be a separated, generatized uniform structure on a set $\mathbb{S}$, and let $\mathscr{P}$ be the associated proximity structure (or proximity class). Then the generalized uniform space $(\mathcal{S}, \mathcal{U})$ admits a completion determined uniquely up to a uniform homemomorphism. Moreover, the proximity space $(\hat{S}, \hat{\mathcal{P}})$ associated with the uniform space $(\hat{\mathcal{S}}, \hat{\mathcal{Y}})$ of the completion, may be interpreted as a proximity-subspace of the proximity space $(\bar{S}, \bar{P})$ of the compactification of $(\mathcal{S}, P)$.
Proof. 1. As before, we denote the collection of all regular Cauchyfilters by $\hat{S}$, and we introduce the notation $\widehat{U}$ for the collection of all sets $\hat{V}$ of the form (4.1) where $\nabla$ is an entourage of $\mathcal{~}$. It is easily verified that the requirements (G.U.1), (G.T.3), (G.U.4) are satisfied for $\widehat{\vartheta}$. To Terify (G.U. o), we consider $\hat{A}_{i} \subset \hat{S}, \hat{C}_{i} \in \widehat{U l}, i=1,2, \ldots, n$. First we choose $\hat{V}_{i} \epsilon \widehat{U}$ such that $\nabla_{i}{ }_{i} \subset U_{i}$ and hence ${\widehat{V^{3}}}_{i} \subset \hat{V}_{i}$ for $i=1,2, \ldots, n$. Since the requirement (G.U.5) is known to be satisfied for the original uniform structure, there must exist an entourage $W$ of $\mathcal{U}$ such that

$$
W\left(\nabla_{i}\left[\hat{A}_{i}\right]\right) \subset \nabla_{i}\left(\nabla_{i}\left[\hat{A}_{i}\right]\right), \quad i=1, \ldots, n .
$$

By the lemma this entails

$$
\widehat{W}\left(\hat{A}_{i}\right) \subset \widehat{V}^{3}\left(\hat{A}_{i}\right) \subset \hat{U}\left(\hat{A}_{i}\right), \quad i=1, \ldots, n
$$

and hence (G.U.5) is satisfied.

Thus we have proved that $\widehat{x \ell}$ is a base of entourages for a generalized uniform structure on $S$.

As usual we define the embedding function $\xi$ of $S$ into $S$ by writing $\xi(x)=\mathcal{F}_{x}$, where $\mathcal{F}_{x}$ denotes the neighbourhood-filter of $x$. Now the uniform bicontinuity of $\xi$ and the density of $\xi(S)$ in $\hat{S}$ can be proved just as in the case of proper uniform structures ([4], p. 163, [2], p. 99). Finally, the uniqueness follows from Theorem 7.

2. We know that the set $\bar{S}$ of the compactification of $(S, P)$ may be interpreted as the set of all maximal regular filters, Cauchy or not (cf. [2], p. 102). Hence $S \subset \bar{S}$. We also recall that the embedding function of the compactification coincides with the function $\xi$ defined above.

Let $\widetilde{\mathcal{U}}$ be the generalized uniform structure induced on $\mathcal{S}$ from the (unique) uniform structure on $(\bar{S}, \bar{P})$, and let $\widetilde{\mathcal{P}}$ be the proximity structure induced on $S$ from $(\bar{S}, \bar{P})$. We recall that the sets $\hat{V}$ where $V$ denotes entourages of the coarsest uniform structure $\chi_{\omega}$ compatible with $\mathscr{P}$, form a base of entourages of $\tilde{\mathcal{U}}$ (cf. [2], p. 101). From this we immediately conclude that $\tilde{\mathscr{U}}$ is coarser than $\hat{\mathscr{U}}$, and hence $\widetilde{\mathcal{P}}$ is coarser than $\hat{\mathcal{P}}$.

To prove the converse, we consider a subset $\hat{A}$ of $\hat{S}$ and an entourage $\hat{U}$ of the form (4.1) of the structure $\hat{\mathcal{C}}$. Let $\hat{V}$ be some entourage of the same form such that $\widehat{V^{3}} \subset \hat{U}$.

From the relation

$$
\nabla[\hat{A}] \Subset V(\nabla[\hat{A}])(\mathscr{P})
$$

we conclude that there exists an entourage $W$ of $\mathcal{U}_{\infty}$ such that:

$$
W(\nabla[\hat{A}]) \subset \nabla(\nabla[\hat{A}]) .
$$


Hence we may apply the lemma once more, giving

$$
\widehat{W}(\hat{A}) \subset \widehat{3}(\hat{A}) \subset \hat{U}(\hat{A}) .
$$

From this we conclude that $\widetilde{\mathcal{P}}$ is finer than $\hat{\mathcal{P}}$, and so the proof is accomplished.

From the second part of Theorem 8 we conclude:

CoRollary. If $\mathcal{P}$ is a $p$-class on $S$, then for every $U \in \mathcal{P}$ the canonical embedding $\xi$ of $(\mathcal{S}, \mathcal{U})$ into the completion $(\hat{S}, \hat{\mathcal{U}})$ will be proximity-homeomorphism of $(S, P)$ into the proximity space $(\hat{S}, \hat{\mathcal{P}})$ associated with $(\hat{S}, \hat{\mathcal{U}})$.

In the paper [12], Yu. M. Smirnov has defined the concept of completeness for proximity spaces. His definition is based on the "uniform $\delta$-coverings" which correspond to the "entourage-like sets" in our theory. It actually requires convergence of every filter which is a Cauchy-filter with respect to all the uniform structures defined by the defining sequence $\left\{\nabla_{n}\right\}_{n=1,2, \ldots}$ of an entourage-like set (cf. part 2 of the proof of Theorem 4).

A completion of a proximity space may then naturally be defined as a $p$-homeomorphic embedding onto a dense subspace of a complete proximity space.

Our next results show that the theory of completion of proximity spaces is covered by the theory of completion of generalized uniform spaces.

From Theorem 3 we immediately get the following:

Proposition 4. Let $\mathcal{P}$ be a proximity structure on $S$, and $\mathcal{U}_{a}$ the finest generalized uniform structure compatible with $\mathcal{P}$. Then $(S, \mathscr{P})$ is a complete proximity space if and only if $\left(\mathcal{S}, \mathcal{U}_{a}\right)$ is a complete generalized uniform space.

THEOREM 9. Let $(S, P)$ be a proximity space, let $U$ be some generalized uniform structure compatible with $\mathcal{P}$, let $\xi$ be the canonical embedding of $(S, \mathcal{U})$ into the completed space $(\hat{S}, \hat{\mathcal{U}})$, and let $\hat{\mathcal{P}}$ be the $p$-structure on $\hat{S}$ assooiated with $\mathcal{U}$. Then $(\xi, \hat{S}, \hat{P})$ is a completion of the proximity space $(S, P)$. In fact every completion of $(S, \supset)$ can be obtained in this way by a suitable choice of $\mathcal{U}$.

Proof. 1. Let $\hat{\mathscr{U}}_{a}$ be the finest generalized uniform structure compatible with $\hat{\mathscr{P}}$. Since $\hat{\mathscr{U}}_{a}$ is finer than $\hat{\mathscr{U}}$, it will possess less regular Cauchyfilters, and so $\left(\hat{S}, \hat{U}_{\alpha}\right)$ is also complete. Hence $(\hat{S}, \hat{\mathscr{P}})$ is a complete proximity space, and $\xi$ will be a proximity homeomorphism in virtue of the Corollary to Theorem 8 .

2. Let $(\xi, \hat{S}, \hat{P})$ be some completion of $(S, P)$, and let $\hat{\mathscr{U}}_{a}$ be the finest generalized uniform structure compatible with $\hat{\mathscr{P}}$. We define the inverse image of $\hat{\mathcal{U}}_{\alpha}$ by $\xi$ (the "S-restriction" of $\hat{\mathcal{U}}_{\alpha}$ ) in the usual way and denote it $\chi$. Let $P^{\prime}$ be the proximity structure associated with $\vartheta$. Br definition, $\xi$ is a uniform homeomorphism relatively to $\mathcal{U}$ and $\hat{\mathcal{U}}_{\alpha}$. Hence it follows from the statement (3) of Corollary 2 to Theorem 6 that $\xi$ is a proximity homeomorphism relatively to $\mathcal{P}^{\prime}$ and $\hat{\mathcal{P}}$. However, $\xi$ is by definition a proximity-homeomorphism relatirely to $\mathcal{P}$ and $\hat{\mathcal{P}}$, and so $\mathcal{P}^{\prime}=\mathcal{P}$. Thus we hare proved that $\mathcal{U}$ is compatible with $\mathcal{P}$. (Note that $\chi$ need $n o t$ be the finest structure $\mathcal{U}_{a}$ compatible with $\mathcal{P}$.) Now $\xi$ is a uniformly bicontinuous mapping of $(S, \mathcal{X})$ onto a dense subset of $\left(\hat{S}, \hat{\mathscr{\ell}}_{a}\right)$, and by Proposition 4 the latter space is complete. Hence $\ell$ is a generalized uniform structure with the desired properties.

COROLlARY. For every completion $(\xi, \hat{\mathcal{S}}, \hat{\mathcal{P}})$ of a proximity space $(\mathcal{S}, \mathcal{P})$ we may interprete $(\hat{S}, \hat{P})$ as proximity subspace of the proximity space $(\bar{S}, \bar{P})$ of the compactification of $(S, P)$. In particular $(S, P)$ admits a maximal completion determined by the coarsest structure $\chi_{\omega}$ compatible with $\mathcal{P}$, and in this aase the extended space equals $(\bar{S}, \bar{P})$ itself. Similarly $(S, \mathcal{P})$ admits a minimal completion determined by the finest structure $\varkappa_{a}$ compatible with P.

Proof. Application of Theorem 8, 9 and the fact that coarser structures within the same class possesses more regular Cauchy-filters (cf. also Theorem 3 of [2], p. 103).

The minimal completion described in the Corollary, was studied by Smirnov, without the use of our generalized uniform structures [12], [15]. He termed it the completion of the given proximity space.

Appendix. In his work [11], p. 562, Yu. M. Smirnov has applied and axiomatic foundation of uniform structures different from that of A. Weil, on which the present paper has been based. It is not difficult to modify Smirnov's axioms such as to define generalized uniform structures instead of proper uniform structures. One only has to replace the "intersection axiom", stating that the product $\alpha \wedge \beta$ of any two coverings $\alpha, \beta$ in the fundamental system $\Gamma$ of coverings again shall belong to $\Gamma$, by the less restrictive requirement that the product $\alpha \wedge \beta$ of any two finite coverings $\alpha, \beta$ in $\Gamma$ again shall belong to $\Gamma$. The proof that this modified system of axioms really defines generalized uniform structures is not essentially different from the equivalence proof in Smirnov's paper [11], p. 572. (By Smirnov this proof is ascribed to A. Kočetkor.)

\section{References}

[1] E. M. Alfsen, J. E. Fenstad, On the equivalence between proximity structures and totally bounded uniform structures, Math. Scand. J. 7(1959), pp. 353-360.

[2] - - A note on completion and compactification, Math. Scand. 8 (1960), pp. 97-104.

Fundamenta Mathematicae, T. LII 
[3] E. M. Alfsen, J. E. Fenstad, Correction to a paper on proximity and totally bounded uniform structures, Math. Scand. 9 (1961), p. 258.

[4] N. Bourbaki, Topologie générale, Chapters I-II, Act. Sci. et Ind., pp. 8581142 , Paris 1951 .

[5] - Topologie générale, Chapter IX, Act. Sci. et Ind., p. 1045, Paris 1958.

[6] V. E. Efremovič, The geometry of proximity I, Mat. Sbornik N. S. 31 (1952), pp. 189-200. (in Russian.)

[7] S. Leader, On clusters in proximity spaces, Fund. Math. 47 (1959), 205-213.

[8] - On completion of proximity spaces by local clusters, Fund. Math. 48 (1960), pp. 201-216.

[9] S. Mrówka, On complete proximity spaces, Doklady Akad. Nauk. SSSR, N. S. 108 (1956), pp. $587-590$.

[10] - On the notion of completeness in proximity spaces; Bull. Acad. Polon. Sei., III, 4 (1956), pp. 477-478.

[11] Yu. M. Smirnow, On proximity spaces, Mat. Sbornik, N. S. 31 (1952), pp. 543-574. (in Russian.)

[12] - On completeness of proximity spaces, Doklady Akad. Nauk. SSSR, N. S. S8 (1953), pp. 761-764. (in Russian.)

[13] - On completeness of proximity spaces $I$, Trudy Moskor. Obsc. 3 (1954), pp. 271-306. (in Russian.)

[14] - On completeness of proximity spaces II, Trudy Moskov Obsc. 4 (1955), pp. 21-435. (in Russian.)

[15] - On completeness of uniform spaces and proximity spaces, Doklady Akad. Sci SSSR N. S. 91 (1953), pp. 1281-1284. (in Russian.)

[16] A. Weil, Sur les espaces à structures uniformes et sur la topologie générale, Paris 1937 .

Reşu par la Rédaction le 13. 11. $196 \dot{1}$

\section{Totality of uniform structures with linearly ordered base}

by

\section{E. M. Alfsen and O. Njåstad (Oslo)}

According to [2] a uniform structure is said to be total if it is the finest member of its $p$-equivalence class, i.e. if it is finer than any other uniform structure with the same uniform set-neighbourhoods. (The above form of the definition, specialized to proper uniform structures, is the third characterization of totality in Theorem 3 of [2]. For the notion of $p$-equivalence, ef. also [1], p. 97.)

Yu. M. Smirnov has proved that every metrizable uniform structure is total [5], p. 570 (cf. also [2] Theorem 4). In the present paper we prove that every uniform structure with a linearly ordered base is total. The method of proof is a transfinite extension of a technique which goes back to Efremovič (proof of Lemma 1 of [4], p. 190).

LEMMA 1. Every well-ordered set $B$ contains a cofinal, well ordered subset $C$ such that every proper segment of $C$ has strictly smaller power than $C$ and every cofinal subset of $C$ has the same power as $C$.

Proof. Let $\chi$ be the smallest cardinal of any cofinal subset of $B$. If $\chi=1$, then $B$ contains a last element $b$, and we shall be through with $C=\{b\}$.

For $\chi>1$ the set $I$ of ordinals of cardinality strictly less than $\psi$ contains non-void proper segments $I_{\alpha}=\{\beta \mid \beta \in I, \beta<\alpha\}$, and $I_{\alpha}$ has cardinal strictly less than $\chi$ for every $\alpha \in I$, whereas $I$ itself has cardinal $\%$.

Let $\psi$ be some one-one mapping of $I$ into a cofinal subset of $B$. The set $\psi\left(I_{a}\right)$ can not be cofinal in $B$ for any $\alpha \in I$ since it has cardinal strictly less than $\chi$. Let $\varphi(\alpha)$ denote the least upper bound of $\psi\left(I_{a}\right)$ in $B$ for every $a \in I$. Clearly $\varphi$ is a non-decreasing mapping of $I$ onto a cofinal subset $C=\varphi(I)$ of $B$. Moreover, $C$ is well ordered (in the ordering induced from $B$ ), since it is the image of the well-ordered set $I$ by the non-decreasing mapping $\varphi$. The cardinal of $C$ cannot exceed $\chi$ since $C=\varphi(I)$; hence by the cofinal nature of $C$ it must equal to $\chi$

Now we assume that $D$ is some proper segment of $C$. It follows from the non-decreasing nature of $\varphi$ that $F=\varphi^{-1}(D)$ is a proper segment of $I$. Hence the cardinal of $F$, and hence also of $D=\varphi(F)$, must be strictly less than $\chi$. Thus we have proved that the cofinal well-ordered subset $C$ 